\title{
CARACTERIZACIÓN FISIOLÓGICA DE CEPAS de Bacillus spp. AISLADAS DE LA RIZÓSFERA DE PAPA (Solanum tuberosum)
}

\section{PHYSIOLOGICAL CHARACTERIZATION OF Bacillus spp. STRAINS FROM POTATO (Solanum tuberosum) RHIZOSPHERE}

\author{
Pamela Calvo ${ }^{1}$ y Doris Zúñiga ${ }^{2}$
}

\begin{abstract}
Resumen
Bacillus es un género altamente presente en la rizósfera de diversos cultivos debido a su capacidad de formación de esporas que le da una ventaja de supervivencia en la rizósfera vegetal. En el presente estudio se realizó una descripción morfológica de las colonias, caracterización fisiológica y bioquímica a 43 aislamientos de Bacillus spp. provenientes de la rizósfera del cultivo de papa de dos zonas altoandinas en Perú. Se encontró que la mayoría de estos aislamientos pertenecían al grupo de "Bacillus subtilis". En cuanto a las pruebas fisiológicas de crecimiento a bajas temperaturas $\left(4^{\circ} \mathrm{C}, 10^{\circ} \mathrm{C}, 15^{\circ} \mathrm{C}\right.$ y $\left.20^{\circ} \mathrm{C}\right)$, de las 43 cepas probadas solo una creció a $4^{\circ} \mathrm{C}$, sin embargo, el $98 \%$ creció a $10^{\circ} \mathrm{C}$ y el $100 \%$ a $15{ }^{\circ} \mathrm{C}$ y a $20^{\circ} \mathrm{C}$. Por otro lado, respecto al $\mathrm{pH}$, se observó que $100 \%$ de las cepas, crecieron bien en ambos pHs (4 y 5.5), lo que indica una buena adaptación del crecimiento a pH ácidos. La evaluación de los niveles de crecimiento tanto a bajo $\mathrm{pH}$ y temperatura baja en el tiempo, revelan un patrón bifásico que se explicaría por pequeños periodos de retraso del crecimiento en condiciones de estrés. Debido a las diferencias de adaptación fisiológica entre cepas de la misma especie se puede sugerir la existencia de una gran diversidad intraespecifica de los Bacillus aislados. Asimismo se encontraron correlaciones entre zonas de aislamientos con temperaturas de suelo más frías y las cepas con mejores niveles de crecimiento a $20^{\circ} \mathrm{C}$.
\end{abstract}

Palabras clave: papa, rizósfera, Bacillus spp., pH, temperatura, diversidad

\begin{abstract}
Bacillus is an abundant genus in the rhizosphere of many crops due to its spore formation capacity that gives it an advantage to survive in the rhizosphere. In the present study we worked with 43 Bacillus spp. previously isolated from potato rhizosphere of two highland zones in Peru. We described the morphological characteristic of their colonies and tested them for their physiological and biochemical characterization. Almost all Bacillus strains belong to the "Bacillus subtilis" group. Also acid $\mathrm{pH}$ test plate (4 and 5.5) and low temperature plate test $\left(4^{\circ} \mathrm{C}, 10^{\circ} \mathrm{C}, 15^{\circ} \mathrm{C}\right.$ and $20^{\circ} \mathrm{C}$ ) were done. Of the 43 tested strains only one grew at $4^{\circ} \mathrm{C}$; therefore, $99 \%$ grew at $10^{\circ} \mathrm{C}$, $100 \%$ grew at $15^{\circ} \mathrm{C}$ and $20^{\circ} \mathrm{C}$. On the other hand, $100 \%$ of the strains grew at $\mathrm{pH} 4$ and 5.5 , which indicates an adaptation to acid $\mathrm{pH}$. The evaluation of the growth levels at low $\mathrm{pH}$ and low temperature in time reveals a two-phase pattern that could be explained by small lag periods in stress conditions. Due to the differences in the physiological adaptation among strains of the same species; the occurrence of a great intraspecific diversity of Bacillus isolated from potato crop rhizosphere can be suggested. We also found correlations between low temperatures in the isolations zones and a better growth level at $20^{\circ} \mathrm{C}$ in the plates tested.
\end{abstract}

Key words: potato, rhizosphere, Bacillus spp., $\mathrm{pH}$, temperature, diversity

\section{Introducción.}

Las poblaciones de bacterias en el suelo no se distribuyen al azar. Factores como la composición de los suelos, la materia orgánica, el pH, el agua y la disponibilidad de oxígeno, junto con la planta huésped, desempeñan un papel importante (Alexander, 1980). La concentración de bacterias por gramo de suelo que se haya alrededor de las raíces de las plantas en la llamada rizósfera es mucho mayor que en el resto del suelo (Lynch, 1990), esto se puede deber a los altos niveles de nutrientes que se hayan en la zona que rodea a las raíces que permiten el desarrollo de poblaciones microbianas (Glick, 1995). La rizósfera ha sido definida en 1904 por Hiltner, como el volumen de suelo influenciado por la presencia de raíces de una planta viva, cuya extensión puede variar de acuerdo al tipo de suelo, la especie de planta, su edad y otros factores (Foster, 1998; Killian et al., 2001). En la rizósfera la interacción entre las bacterias y las raíces de las plantas puede ser beneficiosa, en 
este caso se puede considerar la rizósfera como una zona de amortiguamiento microbiológico en donde la microflora sirve de protección a la planta del ataque de patógenos (Krupa \& Dommergues, 1981).

En un trabajo previo (Calvo et al., 2008) se realizó el estudio de la diversidad microbiana en la rizósfera del cultivo de papa en los departamentos de Huancavelica y Puno. Se encontró que las bacterias del género Bacillus constituían un grupo importante. Este género de bacterias Gram positivas tienen la ventaja de poseer diversos mecanismos para asegurar su sobrevivencia ante condiciones físicas desfavorables, bajo estas condiciones Bacillus spp. inicia una serie de respuestas; si estas respuestas fallan para poder mantenerse en estado vegetativo se induce la esporulación (Petersohn et al., 2001). La habilidad de las especies de Bacillus de formar endosporas altamente resistentes les da una ventaja competitiva muy importante en un ambiente como el suelo (Stephens, 1998). Asi mismo, Bacillus spp. también se deben adaptar a cambios bruscos de temperatura, para esto cuentan con genes de shock térmico inducibles que incluyen proteínas chaperonas y proteasas (Petersohn et al., 2001). Esta capacidad de ser metabólicamente muy diversos les permite tener una colonización exitosa en el ambiente rizosférico. Entre algunos mecanismos promotores de crecimiento identificados en el género Bacillus, se encuentran la solubilización de fosfato (Chatli et al., 2008), la síntesis de fitohormonas como el ácido indol acético (Vessey, 2003) y la capacidad de controlar algunos hongos patógenos en la rizósfera (Larrea , 2001).

El objetivo del presente trabajo fue caracterizar fisiológicamente el crecimiento de Bacillus spp. en condiciones de $\mathrm{pH}$ bajo y diferentes temperaturas bajas, que son condiciones características de las zonas altoandinas donde crece el cultivo de papa y de cuyas rizósferas fueron aisladas las diferentes cepas. Asimismo se realizaron correlaciones entre morfología de colonia, lugar de procedencia, crecimiento a temperatura baja y a $\mathrm{pH}$ bajo para llegar a conocer la existencia o ausencia de una relación que nos lleve a un mejor entendimiento del comportamiento de este genero.

\section{Materiales y métodos.}

Cepas Bacterianas.

Se utilizaron 43 cepas de Bacillus spp. aisladas previamente de la rizósfera del cultivo de papa de 17 campos en Huancavelica y Puno (Tabla 1)(Calvo et al., 2008) en los meses de enero y febrero. También se utilizaron cepas tipo de B. cereus, B. pumilus, B. subtilis 168 del cepario del LEMYB Marino Tabusso (UNALM); las dos primeras cepas fueron aisladas de muestras de alimentos y la ultima proporcionada por el Centro de Ciencias Genómicas de la UNAM, México.
Tabla 1. Características físico químicas de los suelos muestreados.

\begin{tabular}{|c|c|c|}
\hline $\begin{array}{l}\text { Características } \\
\text { físico-químicas }\end{array}$ & Puno & Huancavelica \\
\hline $\mathrm{pH}$ (promedios) & $6.25+/-0.13$ & $5.8+/-0.28$ \\
\hline C.E dS/m & 0.46 & 0.58 \\
\hline $\mathrm{CaCO}_{3}(\%)$ & 0.00 & 0.00 \\
\hline M. O (\%) & 2.64 & 6.60 \\
\hline $\mathrm{P}(\mathrm{ppm})$ & 20.195 & 8.40 \\
\hline K (ppm) & 231.04 & 75.00 \\
\hline Arena (\%) & 63.96 & 51.00 \\
\hline Limo (\%) & 27.04 & 41.00 \\
\hline Arcilla (\%) & 9.00 & 8.00 \\
\hline Clase Textural & $\begin{array}{l}\text { Franco } \\
\text { arenoso }\end{array}$ & Franco \\
\hline CIC* (me/100g) & 10.695 & 19.85 \\
\hline $\mathrm{Ca}^{2}(\mathrm{me} / 100 \mathrm{~g})$ & 2.93 & 6.56 \\
\hline $\mathrm{Mg}^{2}$ (me/100g) & 0.635 & 0.84 \\
\hline K (me/100g) & 0.34 & 0.10 \\
\hline $\mathrm{Na}(\mathrm{me} / 100 \mathrm{~g})$ & 0.08 & 0.22 \\
\hline $\mathrm{AI}^{3}+\mathrm{H}(\mathrm{me} / 100 \mathrm{~g})$ & 0.78 & 0.38 \\
\hline Temperatura $\left({ }^{\circ} \mathrm{C}\right)^{*}$ & 10 & $8-9$ \\
\hline Altura(msnm) & 4200 & $3900-4000$ \\
\hline Precipitación (mm) & 650 & 700 \\
\hline Variedades de papa & $\begin{array}{l}\text { Ccompis-Imilla } \\
\text { Negra }\end{array}$ & $\begin{array}{l}\text { Peruanita- } \\
\text { Amarilla }\end{array}$ \\
\hline Numero de campos & 8 & 9 \\
\hline
\end{tabular}

Caracterización morfológica y bioquímica de Bacillus spp.

Se realizó un descripción morfológica de cada una de las cepas de Bacillus spp. seleccionadas en agar Triptona Glucosa Extracto de levadura (TGE) en base a la apariencia de la forma, borde, superficie, tamaño, consistencia, color y elevación de las colonias. Esta descripción se realizó después de 48 h de crecimiento del cultivo, incubado a $28^{\circ} \mathrm{C}$. Para la identificación bioquímica a nivel de especie se realizaron las siguientes pruebas según Claus \& Berkeley (1986), HPA (2004), Lennette (1987): reducción de nitratos, hidrólisis de almidón, Voges-Proskauer (VP), lecitinasa, fermentación y producción de gas a partir de glucosa, crecimiento a $50^{\circ} \mathrm{C}$, crecimiento a $7 \%$ de $\mathrm{NaCl}$ y crecimiento en anaerobiosis.

Caracterización fisiológica.

Crecimiento a temperaturas bajas.

Se colocaron $5 \mu$ le cada una de las cepas seleccionadas (a una concentración de $10^{8} \mathrm{cel} / \mathrm{ml}$ ) en placas conteniendo agar TGE por duplicado, y se incubaron a $4^{\circ} \mathrm{C}, 10^{\circ} \mathrm{C}, 15^{\circ} \mathrm{C}$ y $20^{\circ} \mathrm{C}$; se evaluó el crecimiento de las cepas durante 15 días para determinar su capacidad adaptativa y se comparó con el crecimiento de la bacteria en una placa control incubada a $28^{\circ} \mathrm{C}$. Se consideró como escala máxima el 

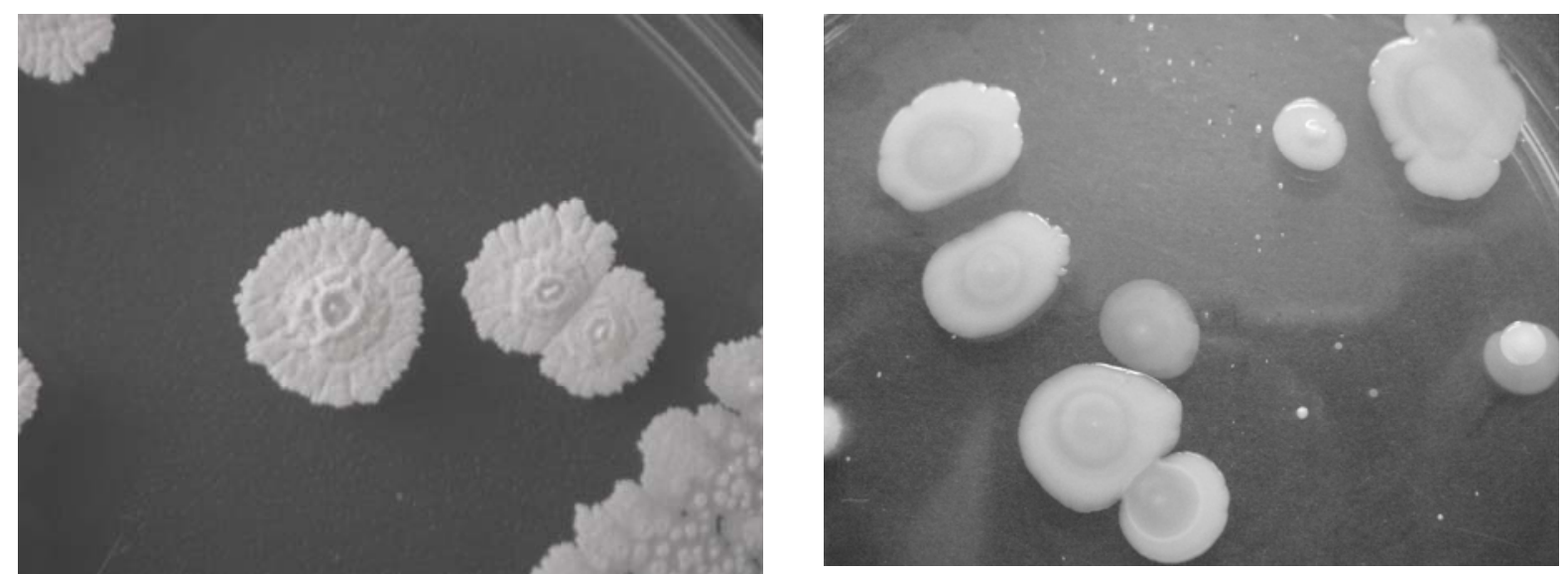

Figura 1. Morfología de diferentes colonias de Bacillus en medio TGE. Colonia con consistencia seca (izquierda). Colonia con consistencia cremosa (derecha).

número 4, que fue el máximo crecimiento de colonia logrado en la placa control. A partir de esto se clasificó el crecimiento de las cepas del 0 al 4.

Crecimiento a pH bajo.

Se colocaron $5 \mu \mathrm{l}$ de cada una de las cepas seleccionadas (a una concentración de $10^{8} \mathrm{cel} / \mathrm{ml}$ ) en placas conteniendo agar TGE con $\mathrm{pH}$ regulado a 4, 5.5 y 7.0. Las pruebas se hicieron por duplicado. El $\mathrm{pH} 7$ se utilizó como control. Las placas se incubaron a $28^{\circ} \mathrm{C}$ por 7 días, al cabo de ese tiempo se evaluó el crecimiento de las cepas para determinar la capacidad adaptativa a suelos de $\mathrm{pH}$ bajo que se presentan en algunas zonas altoandinas. Se utilizó el mismo sistema de evaluación que para la temperatura, basado en una escala del 0 al 4, siendo este último el máximo crecimiento de colonia alcanzado por la placa control. Análisis estadístico.

Se realizó un análisis de correlación de Cramer ( $\mathrm{p}<0.05$ ) (Milton, 2001) con el programa R (R Project, 2008) y el programa SAS (SAS Institute Inc, 1996) para hallar correlaciones entre las diferentes características morfológicas y condiciones de temperatura y $\mathrm{pH}$.

\section{Resultados y discusión.}

Se realizó la descripción de la morfología de las colonias de 43 cepas de Bacillus spp. (Tabla 2). Se observa que una característica común en todas las colonias de Bacillus es su forma irregular y color crema, la apariencia de los bordes de las colonias varía entre aserrada, lobulada, digitiforme y ondulada; las elevaciones de la colonias suelen ser planas o acuminadas, en este último caso presentan el centro más elevado en forma de anillo, la consistencia suele ser seca pero también se presentan colonias cremosas y ligosas (Figura1). La gran mayoría presenta una superficie opaca, y la consistencia y tamaño es variable. Wakita et al. (2007) reportan que las colonias de Bacillus presentan la característica de formar un anillo concéntrico y borde irregular en el centro. Esto es lo que se observó en muchas de las cepas aisladas. Los resultados de las pruebas bioquímicas se muestran en la Tabla 3; el 84\% de las cepas aisladas muestran un comportamiento que las
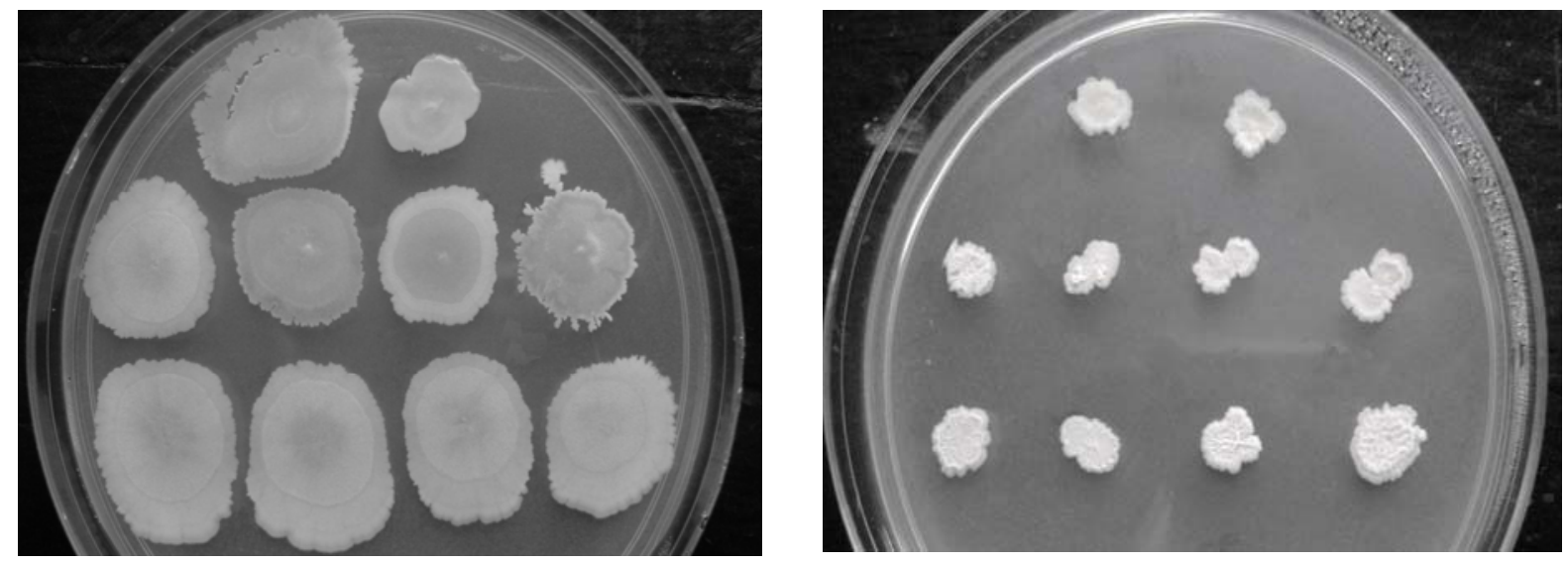

Figura 2. Evaluación de niveles de crecimiento en medio de cultivo a los 15 días. Placa a $28^{\circ} \mathrm{C}$ (control) con nivel de crecimiento máximo de 4 (izquierda). Placa a $10^{\circ} \mathrm{C}$ con nivel de crecimiento 2 (derecha). 


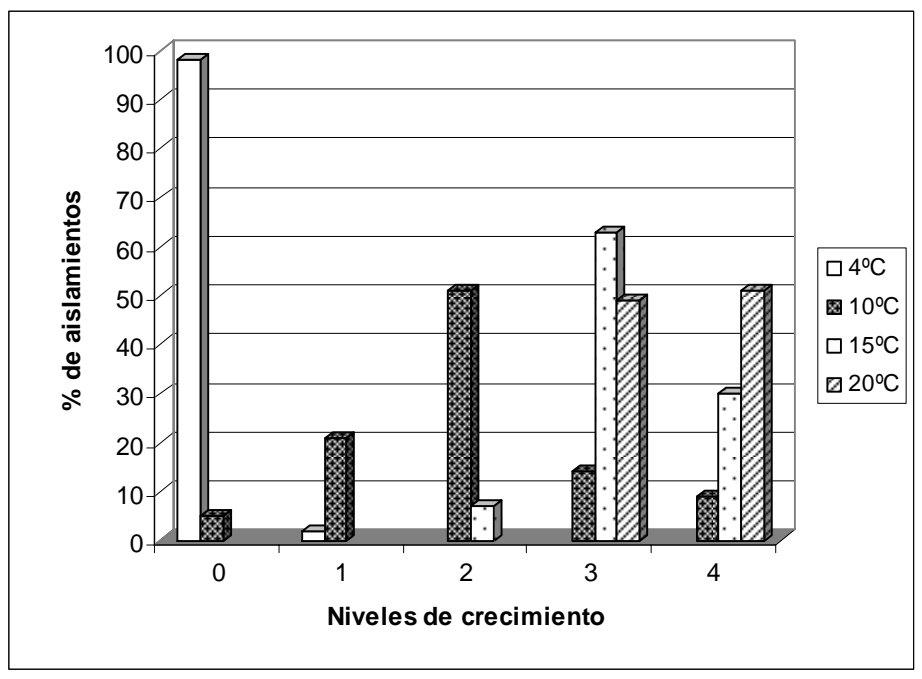

Gráfico 1. Porcentaje de aislamientos en base a 4 niveles de crecimiento en medio de cultivo a bajas temperaturas (15 días) de las cepas de Bacillus spp.

relacionan con la especie $B$. subtilis, las cepas 2, 13 y 25 solo se diferencian de las demás por crecer en anaerobiosis lo que las relaciona más con el género $B$. licheniformis, la cepas 21 y 30 muestran una reacción variable a la prueba de VP, la variabilidad en el resultado de esta prueba ya ha sido reportado por Lennette (1987) por esta razón no se puede afirmar o descartar que pertenezcan al grupo de $B$. subtilis. La
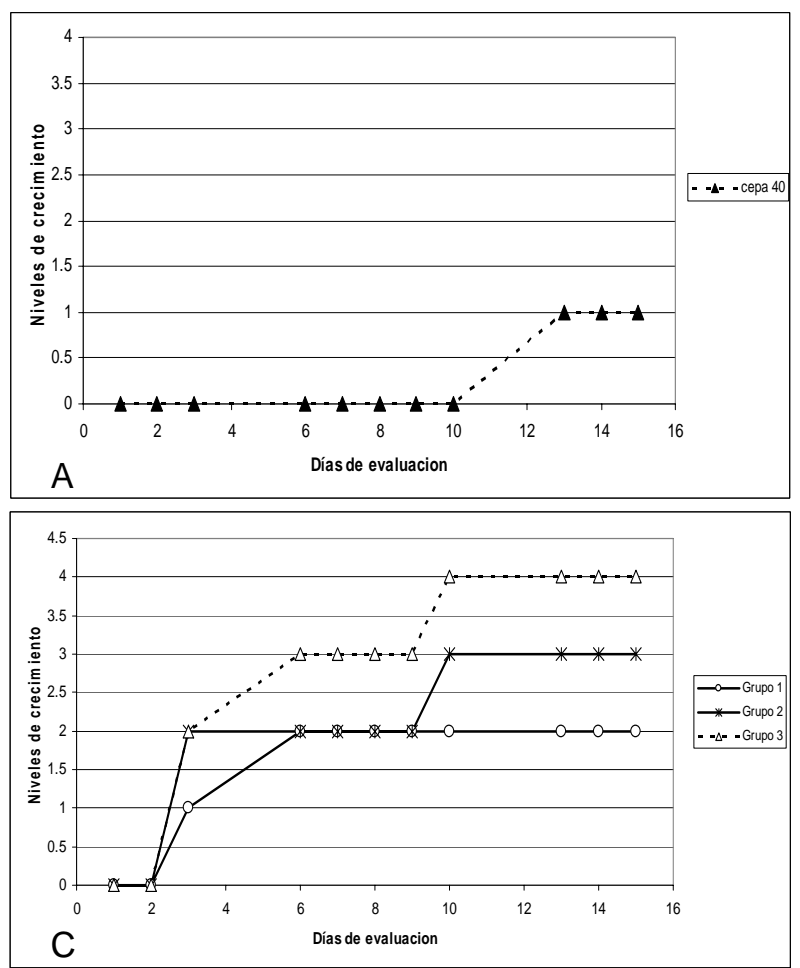

cepa 9 muestra un resultado variable en la producción de acidez en presencia de glucosa, esta prueba no es determinante para la identificación de Bacillus y al igual que la prueba de VP puede presentar este tipo de resultados. Las cepas 28 y 37 no crecen a $50^{\circ} \mathrm{C}$, Claus \& Berkeley (1986) mencionan que la capacidad de crecimiento a $50^{\circ} \mathrm{C}$ es variable entre cepas de la misma especie, los rangos de temperatura de crecimiento altas son muy sensibles tanto que a una variación de un grado puede influenciar en el crecimiento vegetativo de la bacteria. Una de las pruebas más determinantes mencionadas por la HPA (2004) es la prueba de producción de lecitinasa. Esta prueba mide la capacidad de producir esta enzima que degrada la lecitina presente en la yema de huevo. Las 4 principales especies que son lecitinasa positiva son $B$. anthracis, $B$. mycoides, $B$. cereus y $B$. thuringiensis; las tres primeras especies se caracterizan por ser patógenas humanas. En la prueba de lecitinasa realizada ninguna de las cepas aisladas fue lecitinasa positiva, sólo el Bacillus cereus usado como control dio una reacción positiva. Esto es importante ya que en el futuro se podría producir biofertilizante a partir de estas cepas sin correr el riesgo de que sean potencialmente patógenas. $\mathrm{La}$ poca variabilidad de especies aparentemente
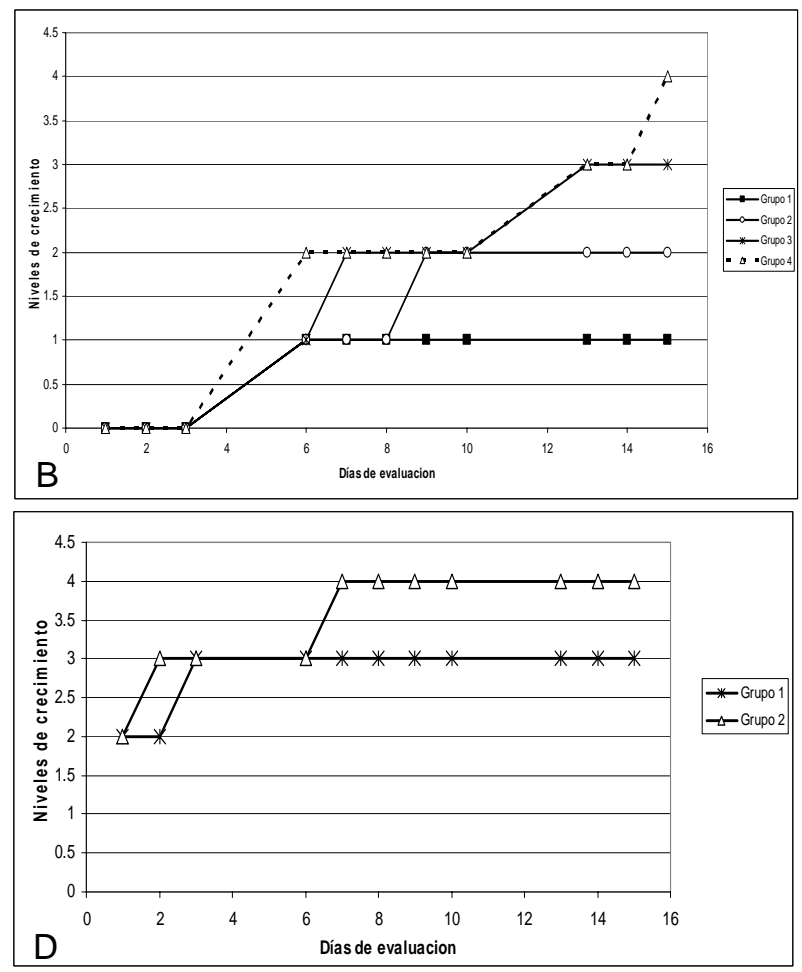

Gráfico 2. Efecto de las temperaturas bajas en el crecimiento de cepas de Bacillus spp. A. Crecimiento a $4^{\circ} \mathrm{C}$. B. Crecimiento a $10^{\circ} \mathrm{C}$. C. Crecimiento a $15^{\circ} \mathrm{C}$. D. Crecimiento a $20^{\circ} \mathrm{C}$. 


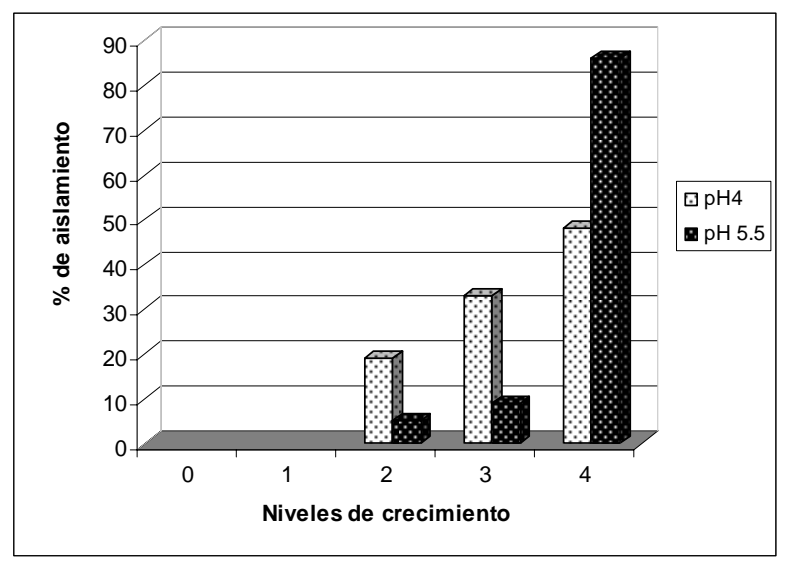

Gráfico 3. Porcentajes de asilamiento en base a los niveles de crecimiento a $\mathrm{pH}$ ácidos en medio de cultivo (7 dias) de las cepas de Bacillus spp.

encontradas no significa necesariamente que la diversidad del suelo sea pobre. McSpadden (2004) menciona que entre las especies mas comúnmente halladas en suelo están $B$. subtilis y $B$. cereus sin embargo, no se ha ratificado que una u otra especie de Bacillus este relacionada más a un tipo de suelo que a otro. Estudios a nivel de ADN han confirmado que la mayoría de las especies de Bacillus que se logran aislar, son aquellas que se caracterizan por ser las mas
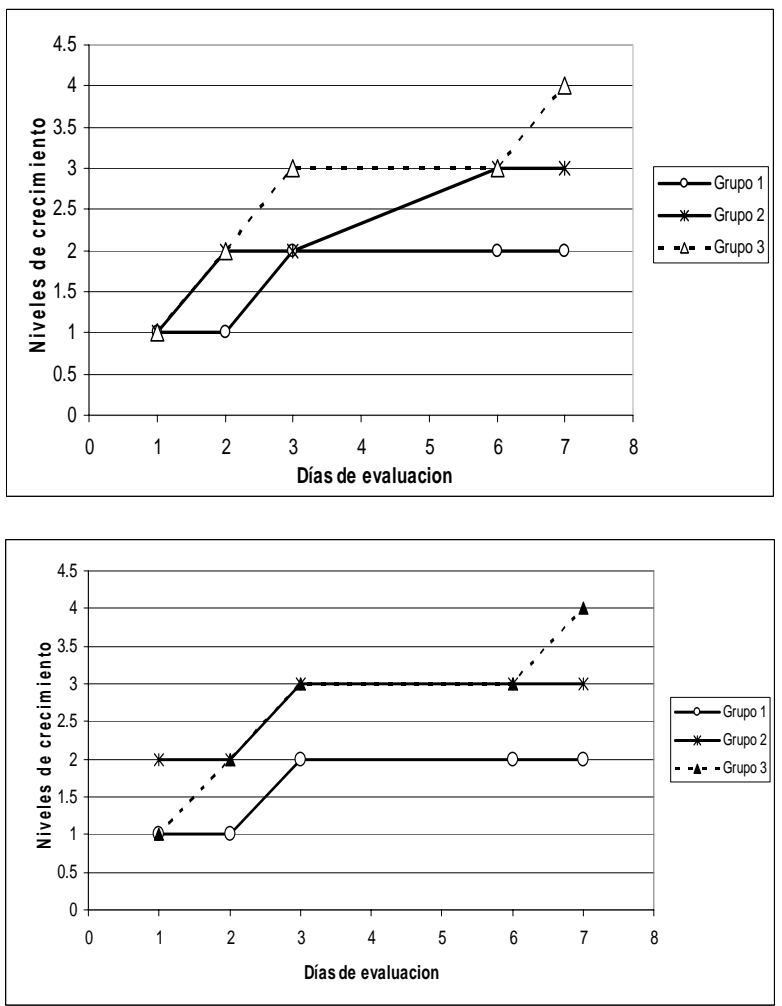

Gráfico 4. Efecto del $\mathrm{pH}$ en el crecimiento de las cepas de Bacillus spp., evaluadas a pH 4 (arriba) y pH 5.5 (abajo). fáciles de cultivar, sin embargo, muchas otras no se descubren por ser difícil la obtención de su cultivo en medios artificiales. Además se debe tener en cuenta que las especies del género Bacillus son genéticamente muy heterogéneas, alcanzando diferencias interespecíficas de un $30 \%$ en la cantidad de G-C (Claus \& Berkeley, 1986).

Las pruebas fisiológicas de temperatura y $\mathrm{pH}$ (Gráficos 2 y 4) indicaron que los porcentajes de cepas que alcanzaron los máximos crecimientos a diferentes temperaturas son muy variables (Gráfico 1), es así que a $4^{\circ} \mathrm{C}$ solo la cepa 40 creció y aún así no alcanzó el máximo nivel de crecimiento. Este comportamiento ya ha sido observado por Ingraham (1958) con bacterias de suelo capaces de crecer incluso a $1^{\circ} \mathrm{C}$ después de un mes. A $10^{\circ} \mathrm{C}$ el porcentaje de cepas que creció fue en aumento pero sólo un $9 \%$ alcanzó el máximo crecimiento. También se observó que un $5 \%$ de las cepas no creció. A $15^{\circ} \mathrm{C}$ todas crecieron y un 30\% alcanzó un crecimiento máximo y a $20^{\circ} \mathrm{C} 51 \%$ alcanzó el máximo nivel de crecimiento 4 y el $49 \%$ el crecimiento en nivel 3. En cuanto al crecimiento en el tiempo en el Gráfico 2 se observa la adaptación de grupos de cepas conformados por cepas con mismos patrones de crecimiento a temperatura baja. Este crecimiento se realizó de manera escalonada, las colonias fueron aumentando de tamaño poco a poco a medida que pasaban los días. A $4^{\circ} \mathrm{C}$ la cepa 40 recién mostró algún crecimiento al día 13 , a $10^{\circ} \mathrm{C}$ las cepas mostraron algún tipo de crecimiento entre los 3 y 6 días y a partir de allí el aumento en el tamaño de la colonia fue escalonado. Las cepas que llegaron al nivel 2 mantuvieron este nivel hasta el final, sin embargo, las que crecieron hasta los niveles 3 y 4 aumentaron poco a poco el tamaño de colonia hasta el último día de evaluación. Este aumento progresivo en el tamaño de colonia podría estar revelando la capacidad de crecimiento que presentan las bacterias a temperaturas bajas. Mientras más baja sea la temperatura, la bacteria tarda más en crecer. En el caso de $B$. subtilis, las temperaturas bajas inducen modificaciones estructurales que se encuentran correlacionadas con patrones de crecimiento atípicos. Se ha observado la existencia de crecimiento celular pero sin división; las bacterias sobreviven, pero por un tiempo no hay división, después de un tiempo de adaptación hay una recuperación y comienza de nuevo la división (Neale \& Chapman, 1970). Por otro lado Méndez et al. (2004) y Budde et al. (2006), mencionan que para Bacillus subtilis las condiciones de baja temperatura inducen en muchos casos los factores de trascripción involucrados en el proceso de esporulación, lo que favorece mas aún el proceso de adaptación.

En el Gráfico 3 se observa que en general, todas las cepas probadas a pH 4 y 5.5 mostraron algún nivel de crecimiento. A pH 4, 48 \% de las cepas llegaron al crecimiento máximo de 4 , mientras que a $\mathrm{pH} 5.5$, el 
88 \% mostró un crecimiento completo en relación al control. Al igual que en el caso de las temperaturas, la capacidad de adaptación aumenta conforme el $\mathrm{pH}$ se acerca al óptimo, Bacillus es un género muy asociada a pH neutros, sin embargo, esta capacidad de adaptación a $\mathrm{pH}$ bajo ya ha sido reportada por $\mathrm{Pal}$ (1998) que menciona que la cepa de Bacillus PAS-2 es capaz de crecer a $\mathrm{pH}$ 4.8. Así también otras cepas aisladas de Bacillus spp. pueden crecer a este $\mathrm{pH}$, pero mostrando diferentes tasas de crecimiento. En el Gráfico 4 se observa que el crecimiento de las cepas también aumenta de manera escalonada a medida que pasan los días, confirmando una vez más su capacidad de crecer en condiciones adversas a través del tiempo.

En el análisis de correlación de Cramer $(\mathrm{p}<0.05)$ se encontró que las cepas con característica de consistencia seca poseen un mejor nivel de crecimiento a $\mathrm{pH} 4$ en medio de cultivo mostrando un coeficiente de correlación de 0.453 . Además, estas mismas cepas presentaron una mejor adaptación a $10^{\circ} \mathrm{C}$ en medio de cultivo, mostrando un coeficiente de correlación de 0.4219. Esto nos indica que la morfología de consistencia seca de la colonia de los Bacillus spp. estaría relacionada con una mejor adaptación a condiciones de estrés.

Por otro lado, a pesar que los valores bajos de $\mathrm{pH}$ no son los valores óptimos de crecimiento para Bacillus, estudios previos llevados a cabo con valores de $\mathrm{pH}$ bajos en suelo (Nishijima et al., 2005) han demostrado la presencia activa de especies de Bacillus.

En cuanto a la procedencia de las cepas (Huancavelica o Puno) y su crecimiento a $20^{\circ} \mathrm{C}$, existe una asociación altamente significativa de 0.674. Las cepas procedentes de Huancavelica llegaron a un nivel máximo de crecimiento de la colonia en medio de cultivo (4), mientras que aquellas aisladas de Puno sólo llegaron a un nivel de crecimiento menor (3). Asimismo la cepa 40 que fue la única que creció a $4^{\circ} \mathrm{C}$ también fue aislada de Huancavelica. Esto nos permite concluir que la adaptación a temperatura baja estaría relacionada con el lugar de procedencia, y las cepas de Huancavelica estarían mejor adaptadas a esas condiciones en comparación a las de Puno. Se realizó también un análisis de correlación entre las temperaturas de los lugares de procedencia de las cepas y el crecimiento a temperatura de $20^{\circ} \mathrm{C}$ en medio de cultivo. En Huancavelica las temperaturas variaron entre 8 y $9{ }^{\circ} \mathrm{C}$ mientras que en Puno se mantuvieron alrededor de $10^{\circ} \mathrm{C}$ (Calvo et al., 2008). Se encontró una correlación significativa con un coeficiente de -0.675 , lo que confirma que las cepas provenientes de zonas de menor temperatura presentan un mejor nivel de crecimiento a $20^{\circ} \mathrm{C}$ en el medio de cultivo.

\section{Conclusiones.}

Se puede concluir que la especie $B$. subtilis es un habitante muy común en la rizósfera del cultivo de papa, que a su vez demuestra una gran diversidad intraespecifica que se confirma con las diferentes capacidades de crecimiento, tanto a temperaturas bajas como a pH ácidos. Es muy importante conocer esta diversidad dada la importancia de la relación no patogénica microorganismo-planta en la rizósfera, donde los microorganismos como Bacillus ejercen una acción especifica mediante la producción de metabolitos secundarios, resistencia a estreses biótico y abióticos y facilitando la toma de nutrientes del suelo; todas estas actividades podrían favorecer el desarrollo del cultivo de papa.

\section{Agradecimientos.}

Dr. Andreas Oswald, Ing. Felipe de Mendiburu del CIP y Bach. Cesar La Torre del LEMYB Marino Tabusso-UNALM. El trabajo ha sido financiado por el Concejo Nacional de Ciencia y Tecnología (Concytec), Centro Internacional de la Papa (CIP), Oficina de Investigación - UNALM y FDA Biol111/UNALM.

\section{Literatura citada.}

Ahmed H., Labuschagne N. \& Korsten L. 2007. Screening rhizobacteria for biological control of Fusarium and crown rot of sorghum in Ethiopia. Biological Control. 40: 97-106.

Alexander M. 1980. Transformaciones microbianas del fósforo. Introducción a la microbiología del suelo. AGT Editor. México. : 355- 371.

Budde I., Steil L., Scharf C., Völker U. \& Bremer E. 2006. Adaptation of Bacillus subtilis to growth at low temperature: a combined transcriptomic and proteomic appraisal. Microbiology. 152: 831-853.

Calvo P., Reymundo L. \& Zúñiga D. 2008. Estudio de las poblaciones microbianas de la rizósfera del cultivo de papa (Solanum tuberosum) en zonas altoandinas. Ecología Aplicada. 7 (1,2): 141-148.

Chatli A.S., Beri V. \& Sidhu B.S. 2008. Isolation and characterisation of phosphate solubilising microorganisms from the cold desert habitat of Salix alba Linn. in trans Himalayan region of Himachal Pradesh. Indian Journal of Microbiology. 48: 267-273.

Claus D. \& Berkeley R.C.W. 1986. Genus Bacillus. En Bergey's Manual of Systematic Bacteriology (Eds. P. H.Sneath, N. Mair, M. E. Sharpe and J.G. Holt, Baltimore, Williams and Wilkins, 9 na. Edición. Vol. 2.: 1105-1139.

Drucker D.B. \& Whittaker D.K. 1971. Microstructure of Colonies of Rod-Shaped Bacteria. Journal of Bacteriology. 108: 515-525.

Foster R.C. 1998. Microenvironments of soil microorganisms. Biology and Fertility of Soils. 6: 189203.

Glick B.R. 1995. The enhancement of plant growth by freeliving bacteria. Canadian Journal of Microbiology. 41: 109-117. 
Health Protection Agency (HPA). 2004. National Standard Method: Identification of Bacillus species. Inglaterra. pp. 7

Hiltner L. 1904. Über neurer erfahrunger und problem auf dem gebeit der bodenbakteriologie und unter besonderer berucksinchtingung der grundungung und brache. Arbeiten Deutscher Landwirtschafts Gesellschaft. 98: 59-78.

Ingraham J.L. 1958. Growth of Psychrophilic bacteria. Journal of Bacteriology. 76: 75-80.

Killian M., Steiner U., Krebs B., Junge H., Schmiedeknecht G. \& Hain R. 2001. FZB24 Bacillus subtilis mode of action of a microbial agent enhancing plant vitality. Pflanzenschut - Nachrichten Bayer. 1: 72-93.

Krupa S.W. \& Dommergues Y.K. 1981. Ecology of root pathogens; 2da. ed. Elsevier scientific publishing company. EEUU.

Larrea O. 2001. Microorganismos antagonistas para el control fitosanitario. Manejo Integrado de Plagas (Costa Rica). 62: 96-100.

Lennette E.H. 1987. Manual de Microbiología Clínica. Editorial médica panamericana. Buenos Aires (Argentina).

Lynch J.M. 1990. The rhizosphere. Willey-Interscience, Chichester, (Inglaterra). : 55-66.

McSpadden B.B. 2004. Ecology of Bacillus and Paenibacillus spp. in Agricultural Systems. Phytopathology. 94: 1252-1258.

Méndez M.B., Orsaria L.M., Philippe V., Pedrido M.E. \& Grau R.R. 2004. Novel Roles of the Master Transcription Factors Spo0A and $\mathbf{U}^{\mathrm{B}}$ for Survival and
Sporulation of Bacillus subtilis at Low Growth Temperature. Journal of Bacteriology. 186: 989-1000.

Milton S. 2001. Estadística para Biología y Ciencias de la Salud. Ed.: McGraw-Hill España. : 407-432.

Neale E.K. \& Chapman G.B. 1970. Effect of low temperature on the growth and fine structure of Bacillus subtilis. Journal of Bacteriology. 104: 518-528.

Nishijima T., Toyota K. \& Mochizuki M. 2005. Predominant culturable Bacillus species in Japanese Arable soils and their potencial as biocontrol agents. Microbes and Environment. 20: 61-68.

Pal S.S. 1998. Interactions o fan acid tolerant strain of phosphate solubilizing bacteria with a few acid tolerante crops. Plant and Soil. 198: 169-177.

Petersohn A., Brigulla M., Haas S., Hoheisel J., Lker U. \& Hecker M. 2001. Global Analysis of the General Stress Response of Bacillus subtilis. Journal of Bacteriology. 183: 5617-5631.

R Project for Statistical Computing Version: 2.7.0. 2008. R Development Core Team.

SAS Institute Inc. 1996. SAS/STAT software: Changes and enhancements for release. 6.12 SAS Institute Inc., Cary, N.C.

Stephens C. 1998. Bacterial sporulation: A question of commitment?. Current Biology. 8: 45-48.

Vessey J.K. 2003. Plant growth promoting rhizobacteria as biofertilizers. Plant and Soil. 255: 571-586.

Wakita J., Shimada H., Itoh H., Matsuyama T. \& Matsushita M. 2001. Periodic Colony Formation by Bacterial Species Bacillus subtilis. Journal of the Physical Society of Japan. 70: 911-919. 
Tabla 2. Descripción morfológica de colonias de Bacillus spp. aisladas de rizósfera de papa.

\begin{tabular}{|c|c|c|c|c|c|c|c|c|}
\hline сера & Forma & Borde & Elevación & Superficie & Color & Consistencia & $\begin{array}{c}\text { Tamaño } \\
(\mathrm{cm})\end{array}$ & Procedencia \\
\hline 1 & Irregular & Aserrado & Plana & Opaca & crema & Muy Ligosa & $0.2-0.3$ & Huancavelica \\
\hline 2 & Irregular & Aserrado & Acuminada & Opaca & crema & Seca & $0.2-0.3$ & Huancavelica \\
\hline 3 & Irregular & Digitiforme & Acuminada & Opaca & crema & Ligosa & $0.3-0.4$ & Huancavelica \\
\hline 4 & Irregular & Digitiforme & Plana & Opaca & crema & Seca & $0.1-0-2$ & Huancavelica \\
\hline 5 & Irregular & Ondulado & Acuminada & Opaca & crema & Poco Ligosa & $0.3-0.4$ & Huancavelica \\
\hline 6 & Irregular & Ondulado & Plana & Brillosa & crema & Cremosa & $0.3-0.4$ & Huancavelica \\
\hline 7 & Irregular & Aserrado & Acuminada & Rugosa & crema & Seca & $0.2-0.4$ & Huancavelica \\
\hline 8 & Irregular & Ondulado & Plana & Opaca & crema & Seca & $0.2-0.3$ & Huancavelica \\
\hline 9 & Irregular & Digitiforme & Acuminada & Opaca & crema & Poco Ligosa & $0.4-0.5$ & Huancavelica \\
\hline 10 & Irregular & Aserrado & Acuminada & Opaca & crema & Poco Ligosa & $0.2-0.3$ & Huancavelica \\
\hline 11 & Irregular & Aserrado & Plana & Opaca & crema & Ligosa & $0.3-0.4$ & Huancavelica \\
\hline 12 & Irregular & Aserrado & Plana & Opaca & crema & Seca & $0.2-0.3$ & Huancavelica \\
\hline 13 & Irregular & Aserrado & Acuminada & Opaca & crema & Seca & $0.3-0.4$ & Huancavelica \\
\hline 14 & Irregular & Aserrado & Acuminada & Opaca & crema & Cremosa & 0.1 & Puno \\
\hline 15 & Irregular & Digitiforme & Pana & Opaca & crema & Seca & $0.1-0.2$ & Puno \\
\hline 16 & Irregular & Ondulado & Acuminada & Opaca & crema & Seca & $0.2-0.4$ & Puno \\
\hline 17 & Irregular & Aserrado & Acuminada & Opaca & crema & Cremosa & 0.1 & Puno \\
\hline 18 & Irregular & Ondulado & Acuminada & Opaca & crema & Seca & $0.1-0.3$ & Puno \\
\hline 19 & Irregular & Ondulado & Acuminada & Opaca & crema & Seca & 0.2 & Puno \\
\hline 20 & Irregular & Digitiforme & Acuminada & Opaca & crema & Seca & 0.2 & Puno \\
\hline 21 & Irregular & Difuminado & Plana & Opaca & crema & Seca & $0.3-0.5$ & Puno \\
\hline 22 & Irregular & Ondulado & Plana & Opaca & crema & Seca & $0.2-0.3$ & Puno \\
\hline 23 & Irregular & Digitiforme & Acuminada & Opaca & crema & Poco Ligosa & $0.2-0.5$ & Puno \\
\hline 24 & Irregular & Ondulado & Acuminada & Opaca & crema & Cremosa & $0.2-0.5$ & Puno \\
\hline 25 & Irregular & Digitiforme & Acuminada & Opaca & crema & Seca & $0.3-0.5$ & Puno \\
\hline 26 & Irregular & Ondulado & Plana & Opaca & crema & Seca & 0.4 & Puno \\
\hline 27 & Irregular & Ondulado & Acuminada & Opaca & crema & Seca & $0.3-0.5$ & Puno \\
\hline 28 & Irregular & Ondulado & Plana & Brillosa & crema & Ligosa & $0.2-0.3$ & Puno \\
\hline 29 & Irregular & Digitiforme & Plana & Opaca & crema & Ligosa & $0.2-0.3$ & Puno \\
\hline 30 & Irregular & Digitiforme & Acuminada & Centro Brilloso & crema & Ligosa & $0.1-0.2$ & Puno \\
\hline 31 & Irregular & Aserrado & Plana & Opaca & crema & Seca & $0.1-0.2$ & Puno \\
\hline 32 & Irregular & Aserrado & Acuminada & Opaca & crema & Seca & $0.1-0.2$ & Puno \\
\hline 33 & Irregular & Ondulado & Plana & Opaca & crema & Seca & 0.1 & Puno \\
\hline 34 & Irregular & Digitiforme & Plana & Opaca & crema & Seca & $0.3-0.4$ & Huancavelica \\
\hline 35 & Irregular & Ondulado & Plana & Opaca & crema & Seca & $0.1-0.2$ & Huancavelica \\
\hline 36 & Irregular & Aserrado & Acuminada & Opaca & crema & Seca & $0.2-0.4$ & Huancavelica \\
\hline 37 & Irregular & Digitiforme & Plana & Opaca & crema & Cremosa & $0.1-0.2$ & Huancavelica \\
\hline 38 & Irregular & Aserrado & Acuminada & Opaca & crema & Seca & $0.1-0.3$ & Huancavelica \\
\hline 39 & Irregular & Ondulado & Acuminada & Opaca & crema & Seca & $0.2-0.4$ & Huancavelica \\
\hline 40 & Irregular & Digitiforme & Acuminada & Opaca & crema & Seca & 0.2 & Huancavelica \\
\hline 41 & Irregular & Ondulado & Acuminada & Opaca & crema & Seca & $0.2-0.4$ & Huancavelica \\
\hline 42 & Irregular & Digitiforme & Acuminada & Opaca & crema & Seca & $0.2-0.4$ & Huancavelica \\
\hline 43 & Irregular & Ondulado & Acuminada & Opaca & Crema & Seca & 0.4 & Huancavelica \\
\hline
\end{tabular}


Tabla 3. Caracterización bioquímicas de 43 cepas de Bacillus spp.

\begin{tabular}{|c|c|c|c|c|c|c|c|c|c|c|}
\hline Cepas & $\begin{array}{c}\text { Voges } \\
\text { Proskauer }\end{array}$ & $\begin{array}{c}\text { Red. } \\
\text { Nitrato }\end{array}$ & Gas & Acidez & $\begin{array}{c}\text { Leciti- } \\
\text { nasa }\end{array}$ & Almidón & $\begin{array}{c}7 \% \\
\mathrm{NaCl}\end{array}$ & $\begin{array}{c}\text { Anaero- } \\
\text { biosis }\end{array}$ & $50^{\circ} \mathrm{C}$ & Especie \\
\hline 1 & + & + & - & + & - & + & + & - & + & \\
\hline 2 & + & + & - & + & - & + & + & + & + & B.licheniformis \\
\hline 4 & + & + & - & + & - & + & + & - & + & B. subtilis \\
\hline 5 & + & + & - & + & - & + & + & - & + & B. subtilis \\
\hline 6 & + & + & - & + & - & + & + & - & + & B. subtilis \\
\hline 9 & + & + & - & $\mathrm{V}$ & - & + & + & - & + & B. subtilis* \\
\hline 10 & + & + & - & + & - & + & + & - & + & B. subtilis \\
\hline 11 & + & + & - & + & - & + & + & - & + & B. subtilis \\
\hline 12 & + & + & - & + & - & + & + & - & + & B. subtilis \\
\hline 13 & + & + & - & + & - & + & + & + & + & B.licheniformis \\
\hline 18 & + & + & - & + & - & + & + & - & + & B. subtilis \\
\hline 19 & + & + & - & + & - & + & + & - & + & B. subtilis \\
\hline 20 & + & + & - & + & - & + & + & - & + & B. subtilis \\
\hline 21 & $\mathrm{~V}$ & + & - & + & - & + & + & - & + & B. subtilis* \\
\hline 22 & + & + & - & + & - & + & + & - & + & B. subtilis \\
\hline 23 & + & + & - & + & - & + & + & - & + & B. subtilis \\
\hline 24 & + & + & - & + & - & + & + & - & + & B. subtilis \\
\hline 25 & + & + & - & + & - & + & + & + & + & B.licheniformis \\
\hline 26 & + & + & - & + & - & + & + & - & + & B. subtilis \\
\hline 27 & + & + & - & + & - & + & + & - & + & B. subtilis \\
\hline 28 & + & + & - & + & - & + & + & - & - & B. subtilis* \\
\hline 29 & + & + & - & + & - & + & + & - & + & B. subtilis \\
\hline 37 & + & + & - & + & - & + & + & - & - & B. subtilis* \\
\hline 38 & + & + & - & + & - & + & + & - & + & B. subtilis \\
\hline 39 & + & + & - & + & - & + & + & - & + & B. subtilis \\
\hline 40 & + & + & - & + & - & + & + & - & + & B. subtilis \\
\hline 41 & + & + & - & + & - & + & + & - & + & B. subtilis \\
\hline 42 & + & + & - & + & - & + & + & - & + & B. subtilis \\
\hline 43 & + & + & - & + & - & + & + & - & + & B. subtilis \\
\hline B.cereus & + & + & - & + & + & + & + & + & - & B. cereus \\
\hline B.subtilis & + & + & - & + & - & + & + & - & + & B. subtilis \\
\hline Bpumilus & + & - & - & + & - & - & + & - & + & B.pumilus \\
\hline
\end{tabular}

Laboratorio de Ecología Microbiana y Biotecnología Marino Tabusso, Dpto. de Biología, Universidad Nacional Agraria La Molina. Av. La Universidad s/n La Molina. pamelacalvovelez@yahoo.com.

2 Laboratorio de Ecología Microbiana y Biotecnología Marino Tabusso, Dpto. de Biología, Universidad Nacional Agraria La Molina. Av. La Universidad s/n La Molina. Email. dzuniga@lamolina.edu.pe, web: www.lamolina.edu.pe/lmt. 